\title{
Miniature loops in the solar corona
}

\author{
K. Barczynski ${ }^{1}$, H. Peter ${ }^{1}$, and S. L. Savage ${ }^{2}$ \\ 1 Max Planck Institute for Solar System Research, Justus-von-Liebig-Weg 3, 37077 Göttingen, Germany \\ e-mail: barczynski@mps.mpg.de \\ 2 NASA Marshall Space Flight Center, ZP 13, Huntsville, AL 35812, USA
}

Received 5 July 2016 / Accepted 4 November 2016

\begin{abstract}
Context. Magnetic loops filled with hot plasma are the main building blocks of the solar corona. Usually they have lengths of the order of the barometric scale height in the corona that is $50 \mathrm{Mm}$.

Aims. Previously it has been suggested that miniature versions of hot loops exist. These would have lengths of only $1 \mathrm{Mm}$ barely protruding from the chromosphere and spanning across just one granule in the photosphere. Such short loops are well established at transition region temperatures $(0.1 \mathrm{MK})$, and we investigate if such miniature loops also exist at coronal temperatures $(>1 \mathrm{MK})$. Methods. We used extreme UV (EUV) imaging observations from the High-resolution Coronal Imager (Hi-C) at an unprecedented spatial resolution of $0.3^{\prime \prime}$ to $0.4^{\prime \prime}$. Together with EUV imaging and magnetogram data from the Solar Dynamics Observatory (SDO) and X-Ray Telescope (XRT) data from Hinode we investigated the spatial, temporal and thermal evolution of small loop-like structures in the solar corona above a plage region close to an active region and compared this to a moss area within the active region.

Results. We find that the size, motion and temporal evolution of the loop-like features are consistent with photospheric motions, suggesting a close connection to the photospheric magnetic field. Aligned magnetograms show that one of their endpoints is rooted at a magnetic concentration. Their thermal structure, as revealed together with the X-ray observations, shows significant differences to moss-like features.

Conclusions. Considering different scenarios, these features are most probably miniature versions of hot loops rooted at magnetic concentrations at opposite sides of a granule in small emerging magnetic loops (or flux tubes).
\end{abstract}

Key words. Sun: corona - Sun: magnetic fields - Sun: UV radiation - Sun: activity - methods: data analysis

\section{Introduction}

The major building blocks of the solar corona are loops. Observations of these structures have existed since the 1940s (Bray et al. 1991), with the key information acquired through extreme UV (EUV) and X-ray observations. Coronal loops cover a wide range of temperatures and lengths; from small transition region loops at $0.1 \mathrm{MK}$ being only a few Mm long (Peter 2001; Hansteen et al. 2014) to loops hotter than $10 \mathrm{MK}$ and/or longer than $100 \mathrm{Mm}$ (Reale 2010). A typical active region loop would have a temperature of approximately $3 \mathrm{MK}$ and a length above $10 \mathrm{Mm}$ (Reale 2010). Naturally, the question appears regarding the possible minimum length of a hot $(>1 \mathrm{MK})$ coronal loop. Magnetic field lines originating from very small bipoles might not reach above the height of the average chromosphere, which is, according to semi-empirical models, at some $2 \mathrm{Mm}$. Assuming a semi-circular geometry of the field line, this would correspond to a footpoint distance of $4 \mathrm{Mm}$ in the photosphere. However, the solar atmosphere is in a dynamic state, therefore one might expect loops even shorter than that.

The presence small bipolar magnetic structures carried upward either by granular convection or magnetic buoyancy was proposed by Lites et al. (1996) in the context of horizontal inter-network magnetic fields. Using spectro-polarimetry Centeno et al. (2007) showed that such low-lying magnetic loops can connect opposite magnetic polarities that are separated by only $2^{\prime \prime}$ in the photosphere. Such small magnetic bipoles can emerge, isolated in a transient fashion (Ishikawa et al. 2008) with a mean lifetime of approximately 4 min (Ishikawa $\&$ Tsuneta 2009). In small-scale emergence processes the separation between the footpoints in the photosphere ranges from $0.5 \mathrm{Mm}$ to $4 \mathrm{Mm}$ and is correlated with the lifetime of the emerging structure (Martínez González \& Bellot Rubio 2009). To reconstruct the three-dimensional structure of the magnetic field during the small-scale mergence, Ishikawa et al. (2010) investigated spectro-polarimetric data to invert the magnetic field vector as a function of height in the atmosphere. They showed that the rising structures are indeed flux tubes with enhanced magnetic field reaching heights of $400 \mathrm{~km}$ above optical depth unity at the surface (their Fig. 8). Their diagnostics were limited to the photosphere, but it seems reasonable to assume that such a flux tube could rise all the way to the top of the chromosphere if the emerging field was strong enough.

Indirect evidence for the existence of small cool transition region loops was first suggested by Feldman (1983) based on spectroscopic data. In particular, he argued that part of the transition region emission originates in unresolved fine structures, which would be cool loops not connected to the corona above. With the help of spectral maps and spectroscopic investigations Warren \& Winebarger (2000) and Peter (2000) investigated the properties of such transition region loops. Because of instrumental limitations, these could not be imaged directly in a clear fashion until the IRIS spectrograph and imager (De Pontieu et al. 2014) became available. Using slit-jaw images, Hansteen et al. (2014) could follow the evolution of such cool loops with lengths of only a few $\mathrm{Mm}$ and lifetimes of a few minutes. Appearing in the quiet Sun network, considering their length, such loops would only span across one granule, probably connecting opposite magnetic polarities in the intergranular lanes. Such small cool transition region loops have also been investigated in 


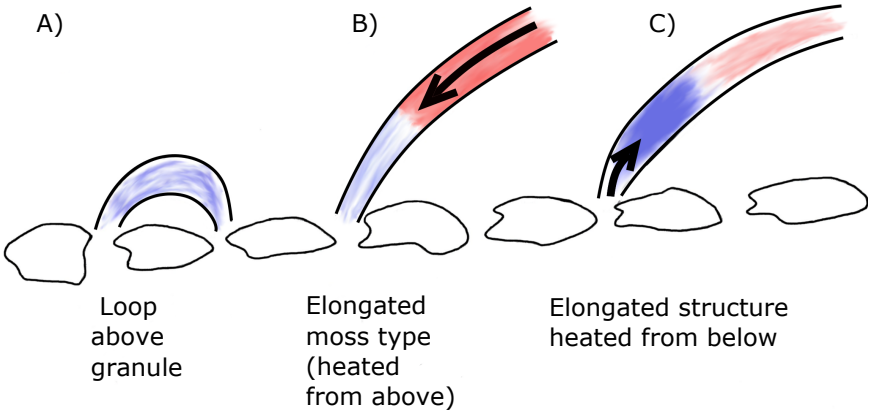

Fig. 1. Three scenarios for miniature loop-like structures seen between $1 \mathrm{MK}$ and $2 \mathrm{MK}$. A) Small loops spanning across one granule, B) short elongated structures at the footpoint of a hot loop, e.g. moss, and C) footpoint segments of a dilute hot loop heated from below (see Sect. 1). The black solid lines indicate granules. The blue and red colours in the loop features denote warm (1 MK to $2 \mathrm{MK}$ ) and hot ( $>5 \mathrm{MK}$ ) plasma. The arrows represent the direction of the energy flux powering the bright feature, that is, in panel B) the heat flux from the corona down to the lower atmosphere and in panel $\mathbf{C}$ ) the Poynting flux into the upper atmosphere.

one-dimensional models. Sasso et al. (2012) showed that loops with lengths of some $1 \mathrm{Mm}$ to $15 \mathrm{Mm}$ could in principle explain the increase of the emission measure towards lower temperatures below $10^{5} \mathrm{~K}$. In their models the quasi-static loops always remained well below $1 \mathrm{MK}$.

With the existence of small cool loops now being firmly established, the question is; can such small loops also reach higher coronal temperatures? One conceptual argument against this would be that a short magnetic fieldline with a length of only one or a few Mm would still be covered by the chromosphere. Because of the high density there, it might be unlikely in terms of energy requirements to heat a significant amount of material to coronal temperatures. However, recent spectroscopic observations have shown evidence of plasma in the dense transition from the photosphere to the chromosphere at approximately the temperature minimum perhaps being heated to almost $100000 \mathrm{~K}$ (Peter et al. 2014). Therefore, it might well be that such structures are further heated to coronal temperatures.

Small elongated structures with a footpoint distance of only $1 \mathrm{Mm}$ reaching more than $1 \mathrm{MK}$ have been reported by Peter et al. (2013). Using data obtained from the High Resolution Coronal Imager (Hi-C; Cirtain et al. 2013), they found these structures to have a width of less than $200 \mathrm{~km}$. These observations were only possible because of the high spatial resolution of the suborbital rocket experiment $\mathrm{Hi}-\mathrm{C}$. Its resolution is approximately five times higher than that of the current workhorse of coronal imaging studies, the Atmospheric Imaging Assembly (AIA; Lemen et al. 2012), which has a spatial resolution of approximately $1.4^{\prime \prime}$ corresponding to $1 \mathrm{Mm}$ and would not show those extremely small features.

The small-scale coronal structures have been interpreted as miniature loops by Peter et al. (2013), spanning one granule (Fig. 1a) and connecting two opposite magnetic field polarities in the intergranular lanes. This would provide a natural explanation for their length, which is comparable to a granule, and be consistent with the emerging bipoles discussed above. Also, the motion of such miniature loops should be of the order of the photospheric horizontal motions which is typically of the order of $1 \mathrm{~km} \mathrm{~s}^{-1}$ (e.g. Dravins 1975). However, Peter et al. (2013) could not exclude an alternative scenario in which these elongated structures would be the moss-type emission (Fig. 1b). Moss emission is characterised by dynamic arcsecond-scale features at the footpoint region of a hot loop typically reaching more than $5 \mathrm{MK}$ and being visible in X-rays (Berger et al. 1999). In some sense the $1 \mathrm{MK}$ to $2 \mathrm{MK}$ emission near the footpoints represents the transition region of that hot loop. In the Hi-C data set Testa et al. (2013) and Morton \& McLaughlin (2014) analysed moss structures but in the more active part of the $\mathrm{Hi}-\mathrm{C}$ fieldof-view, and not in the plage region where Peter et al. (2013) found the small elongated structures. There would also be a third option, where a longer structure is heated from below, filling only the lower part of the loop with $1 \mathrm{MK}$ to $2 \mathrm{MK}$ plasma near the footpoint with a dilute (basically invisible) hot part above (Fig. 1c).

The small structures we report here are quite different from coronal bright points, originally observed by Golub et al. (1974) in X-rays. Those are much bigger with an overall average size of approximately $30^{\prime \prime}$ and a bright core of approximately $5^{\prime \prime}$ to $10^{\prime \prime}$. They have much longer lifetimes of several hours and are typically associated with a bi-polar magnetic feature at the surface. However, there is a (magnetic) substructure in these features (Brown et al. 2001), and it has been suggested that a bright point might consist of small loops with widths of only approximately $1^{\prime \prime}$ to $2^{\prime \prime}$ and temperatures of approximately $1.6 \mathrm{MK}$ (Dere 2008). Nevertheless, it does not seem likely that the tiny loop-like features directly observed by Peter et al. (2013) and investigated here are related to the proposed elementary structures of a coronal bright point. This is supported by the magnetic structure, which is clearly bi-polar for a bright point, but mostly unipolar (perhaps with small-scale non-resolved opposite polarities) for the plage-type region hosting the very small loop-like features.

Our aim is to understand the nature of the tiny elongated structures identified by Peter et al. (2013) and to distinguish between the three scenarios outlined above and sketched in Fig. 1. In particular, we investigate the morphology and evolution (Sect.3), the underlying magnetic field (Sect.4), and the thermal properties (Sect. 5) of these features.

\section{Observations}

In this study we concentrated on data acquired during a suborbital sounding rocket flight of the Hi-C (Cirtain et al. 2013; Kobayashi et al. 2014). It was launched on 11th July, 2012, and acquired data for approximately $5 \mathrm{~min}$ of the active region AR11519-21 and its surroundings. For our analysis, we complement this (Table 1) with data from the X-Ray Telescope onboard Hinode (XRT; Golub et al. 2007) and the Solar Dynamics Observatory (SDO); in particular the EUV images from the Atmospheric Imaging Assembly (AIA; Lemen et al. 2012) and magnetograms from the Helioseismic and Magnetic Imager (HMI; Scherrer et al. 2012).

The EUV imager Hi-C provides data of the solar corona in a $5 \AA$ wide wavelength band around $193 \AA$ dominated by emission from Fe XI originating at approximately $1.5 \mathrm{MK}$. In the context of coronal imaging, $\mathrm{Hi}-\mathrm{C}$ has an unprecedented plate scale of $0.1^{\prime \prime}$ pixel $^{-1}$, which corresponds to $73 \mathrm{~km}$ pixel ${ }^{-1}$ on the Sun. The image resolution estimated by Winebarger et al. (2014) is $0.3^{\prime \prime}$ to $0.4^{\prime \prime}$. The $4 \mathrm{k} \times 4 \mathrm{k}$ full-frame images were recorded with an exposure time of $2 \mathrm{~s}$ at a cadence of $5.5 \mathrm{~s}$. The unprecedented spatial and temporal resolution achieved by the Hi-C allows us to carry out the detailed analysis of the small-scale arcsec-size structures discussed in the introduction that were not resolved by previous instruments. We have studied 25 full-frame images (level-1.5) of the active region obtained between 18:53:11 UT and 18:55:30 UT on 11th July, 2012. All times in this paper are 
Table 1. Imaging data used in this study.

\begin{tabular}{cccc}
\hline \hline & Band & Contribution & $T_{\text {peak }}[\mathrm{MK}]^{c}$ \\
\hline Hi-C & $193 \AA$ & Fe XII & 1.5 \\
\hline & $1600 \AA$ & continuum & $<0.01$ \\
& $131 \AA$ & Fe VIII & 0.4 \\
& $171 \AA$ & Fe IX & 0.7 \\
$\mathrm{AIA}^{a}$ & $193 \AA$ & Fe XII & 1.5 \\
& $211 \AA$ & Fe XIV & 2.0 \\
& $335 \AA$ & Fe XVI & 2.8 \\
& $94 \AA$ & Fe XVIII & 7.1 \\
\hline $\mathrm{XRT}^{b}$ & Ti-Poly & free-free emission & 9 \\
\hline
\end{tabular}

Notes. ${ }^{(a)}$ All listed AIA channels are used to co-align the Hi-C observations with the HMI magnetograms, and all but the $1600 \AA$ channel are employed in the DEM analysis (Sect. 5.1). ${ }^{(b)}$ The XRT observations are used for thermal diagnostics. ${ }^{(c)}$ For the Hi-C and AIA bands, $T_{\text {peak }}$ is the temperature of peak ion fraction of the main contributing ion in the respective band according to O'Dwyer et al. (2010). Most AIA bands have significant contributions from other temperatures as well. The value for XRT is taken from Golub et al. (2007).

in seconds from this first frame. We do not analyse the earlier 11 images (before 18:53:11 UT), because at this point the focus was not yet locked. The images after 18:55:30 contain only a partial read out of the detector $(1 \mathrm{k} \times 1 \mathrm{k})$ acquired at a higher cadence, which unfortunately did not cover the region we are interested in here. The Hi-C images are aligned to sub-pixel level accuracy using a cross-correlation technique to obtain a jitterfree data set.

For context, for thermal studies (Sect. 5.1), and the alignment with the magnetic field data (Appendix B), we used data from various channels of AIA (cf. Table 1). This provided fulldisk images at a plate scale of $0.6^{\prime \prime}$ pixel $^{-1}$ at a cadence of $12 \mathrm{~s}$ or more. In Fig. 2 we show the context of the active region observed by $\mathrm{Hi}-\mathrm{C}$ as well as our regions of interest. In this paper we concentrate on two small plage-type regions that are shown in Figs. $2 \beta$ and $\gamma$ along with areas marked for a more detailed study.

To investigate the relationship between the small loop-like features and the surface magnetic field, we use magnetogram data from HMI. Provided with a plate scale of $0.5^{\prime \prime}$ pixel $^{-1}$ and a temporal cadence of $45 \mathrm{~s}$, HMI provides only a few snapshots during the $140 \mathrm{~s}$ of the full-frame Hi-C data used here. Thus, we concentrate on the line-of-sight magnetogram taken at 18:54:53 UT in the middle of the Hi-C full-frame coverage. The spatial alignment between $\mathrm{HMI}$ and $\mathrm{Hi}-\mathrm{C}$ was achieved through a sequence of AIA channels using a cross-correlation procedure (see Appendix B). We estimate the accuracy of this alignment to be better than $0.2^{\prime \prime}$ (Table B.1), that is, slightly more than half a pixel of HMI. While magnetic field information at higher spatial resolution and magnetic sensitivity than that available with HMI would have been desirable, unfortunately, such data were not available for our region of interest, either from the ground or from the space-based Hinode observatory.

To study the possible presence of very hot plasma in relation to the small loop-like features, we used data from XRT that provides a spatial scale of $1^{\prime \prime}$ per pixel. During the Hi-C flight XRT, data were taken with the Ti-poly filter showing plasma in a broad temperature with a peak at approximately 9 MK. From the peak down to a temperature of $2 \mathrm{MK}$, the response of Ti-poly drops by a factor of approximately 15 (Golub et al. 2007, their
Fig. 7). Unfortunately, the plage area of interest in our study is only partially covered by the XRT field-of-view. Still, the region connecting the plage to the main part of the active region is covered, which makes the XRT data very valuable for our study (see Sect. 5.2)

\section{Properties of small loop-like features}

Small loop-like features have been found in Hi-C observations by Peter et al. (2013). Based on a single image they found that these have a length of approximately $1.5 \mathrm{Mm}$ and a width below $200 \mathrm{~km}$, and they suggest an interpretation of them as miniature versions of coronal loops. The main purpose of our study is to investigate the spatial and temporal evolution and the thermal structure of these features and to relate them to the underlying magnetic field structure. We first discuss length, width and relative orientation with respect to the E-W direction of the Sun and horizontal motions of the loop-like structures as a function of time.

\subsection{Identification of small loop-like features}

Identification of the loop-like features is via a combination of manual and automated procedures. Firstly, on each of the 25 images, we identified the $15^{\prime \prime} \times 15^{\prime \prime}$ subregion hosting the features of interest (Fig. 3). For each image, the intensity in that subregion of $15^{\prime \prime} \times 15^{\prime \prime}$ was normalised by the respective median value of intensity from that region. Then, the contrast of the subregion is enhanced by employing a median filter with a kernel size of $3^{\prime \prime}$.

We approximately identified four features by eye, marked A to D in Fig. 3. For each structure we defined a rectangular subfield only just covering this feature and its immediate surroundings (e.g. box 1 in Fig. 3 for feature A). In each of the small boxes, we calculated the maximum of the normalised and contrast-enhanced brightness, $I_{\max }$, during the time series. We then defined the small loop-like structures as the feature enclosed by the contour line at a level of $90 \%$ of $I_{\max }$ (cf. contours A to D in Fig. 3).

To describe the properties of the features we fitted an ellipse ${ }^{1}$ to the respective contour lines at a level of $90 \%$ of $I_{\max }$. This is motivated by the observation that the contour lines have an approximately ellipsoidal shape. The properties of the fitted ellipse in each time step was then used to characterise the loop-like features: the position of the ellipse represents the position of the loop feature; for its length and width we use the major and minor axis of the ellipse, the angle is defined as the angle of the major axis with solar- $x$, that is, the E-W direction and the brightness of each feature was calculated by the mean brightness within the ellipse. This was done independently for each of the exposures of the time series of full-frame $\mathrm{Hi}-\mathrm{C}$ data so that we could also investigate the temporal evolution, at least over the section covered by more than two minutes of the full-frame data. To study the brightness variability we used images without contrast enhancement by the median filtering, of course.

The spatial extent of the loop-like features, as characterised by the ellipse contours at $90 \%$ intensity level, is a measure for the full width at half maximum of the features. While the plage region shows a peak intensity of a factor of approximately

\footnotetext{
1 For ellipse fitting we use the function mpfitellipse from the IDL library of Craig Markwardt (https : //www . physics . wisc . edu/
} $\sim$ craigm/idl/). 

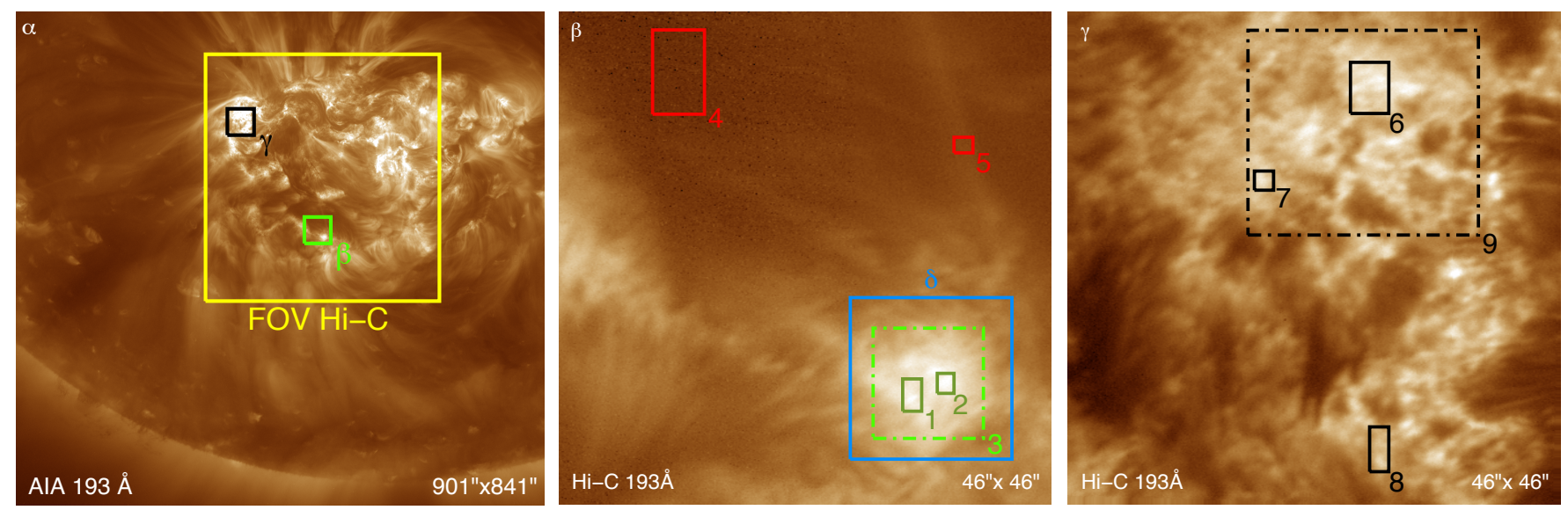

Fig. 2. Active region context and regions of interest. All images are taken in the $193 \AA$ channel showing emission around $1.5 \mathrm{MK}$ (see Table 1 ). Panel $\alpha$ ): part of the solar disk seen in AIA during the Hi-C rocket flight. The full field-of-view of Hi-C is indicated by the yellow box. The green and black boxes $\beta$ and $\gamma$ indicate the plage area and the moss region displayed in panels $\beta$ and $\gamma$, respectively. Panel $\beta$ ): zoom into the plage region south of the active region $\left(46^{\prime \prime} \times 46^{\prime \prime}\right)$. The boxes 1 to 5 indicates features used for the thermal study in Sect. 5.1. The box $\delta$ highlights the field-of view shown in Fig. 3. Panel $\gamma$ ): zoom into a moss region. As in panel $\beta$ ), the numbered boxes show the features for the thermal study.
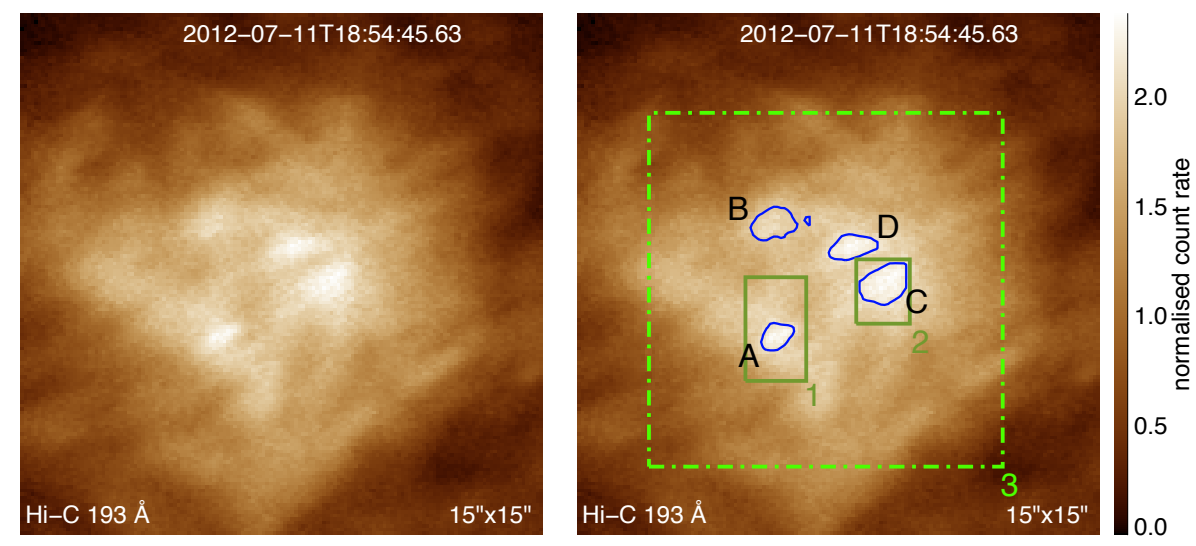

Fig. 3. Zoom of the plage area seen by Hi-C (see box $\delta$ in Fig. 2 $\beta$ ). We show the same image with and without annotation. The contours A to D show the miniature loop-like features identified with the procedure outlined in Sect.3.1. The boxes 1 to 3 show the features for the thermal analysis in Sect. 5.1 (same boxes as in Fig. $2 \beta$ ).

10 higher than the surrounding quiet areas, the individual looplike features have an intensity contrast of approximately $20 \%$ above the plage region in the immediate vicinity of them (see Fig. 4 of Peter et al. 2013). Therefore, the $90 \%$ level of the peak intensity (without background subtraction) represents the full width at half maximum. The values derived for the widths and lengths of the loop-like features can be lower limits only, because the background emission from the plage region might hide a larger (low intensity) extension of the features. However, this argument applies to any measurement of, for example, loop widths in the corona.

\subsection{Properties of small loop-like features}

The position of the features slowly changes in space, typically over slightly less than $1^{\prime \prime}$ over the $140 \mathrm{~s}$ covered by the fullframe images. The positions of the four features and their evolution in time is shown in Fig. 4. To check that there are no systematic residual image motions, we check the mean position (equal weighting) of the four intensity features that is found in the middle of Fig. 4. This is almost stable with a motion of $<0.2^{\prime \prime}$ in $140 \mathrm{~s}\left(\cong 1.0 \mathrm{~km} \mathrm{~s}^{-1}\right)$. This reflects the good temporal alignment of the Hi-C data. The trajectories of the four features (A to D) cover approximately $1^{\prime \prime}$ during the observation in a more or less linear fashion. The motion of $\approx 1^{\prime \prime} / 140 \mathrm{~s}$ corresponds to a speed

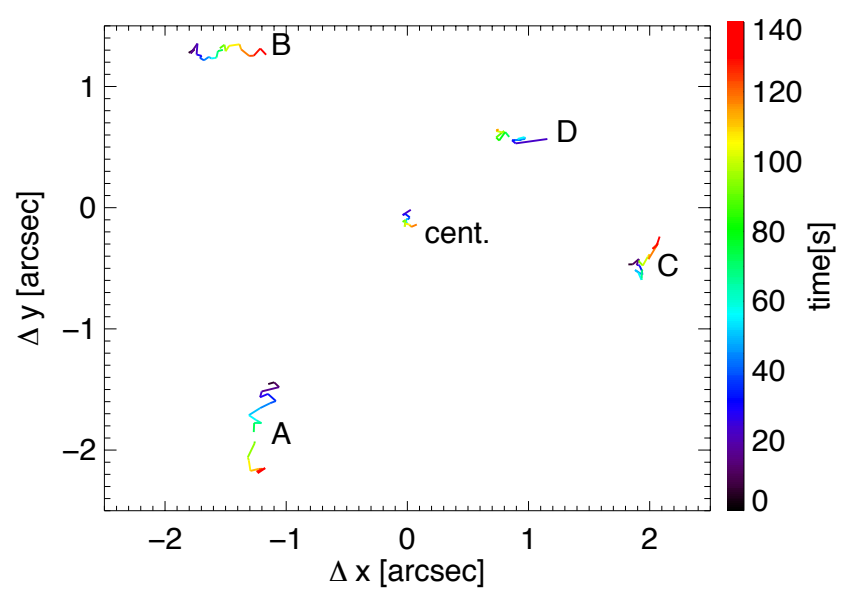

Fig. 4. Horizontal motion of small loop-like features. The trajectories A to $\mathrm{D}$ show the centre of the respective features identified in Fig. 3 as a function of time (colour-coded) over 140 s (after 18:54:11 UT). The error estimate for the position from the ellipse fitting procedure is approximately 0.5 pixels or $0.05^{\prime \prime}$ (half a tick mark). For comparison, the trajectory labelled "cent." shows the mean position of the four loop-like features A to D. See Sect. 3.1.

of $5 \mathrm{~km} \mathrm{~s}^{-1}$. That all four features move in random directions (as far as can be judged from four data points) underlines the 

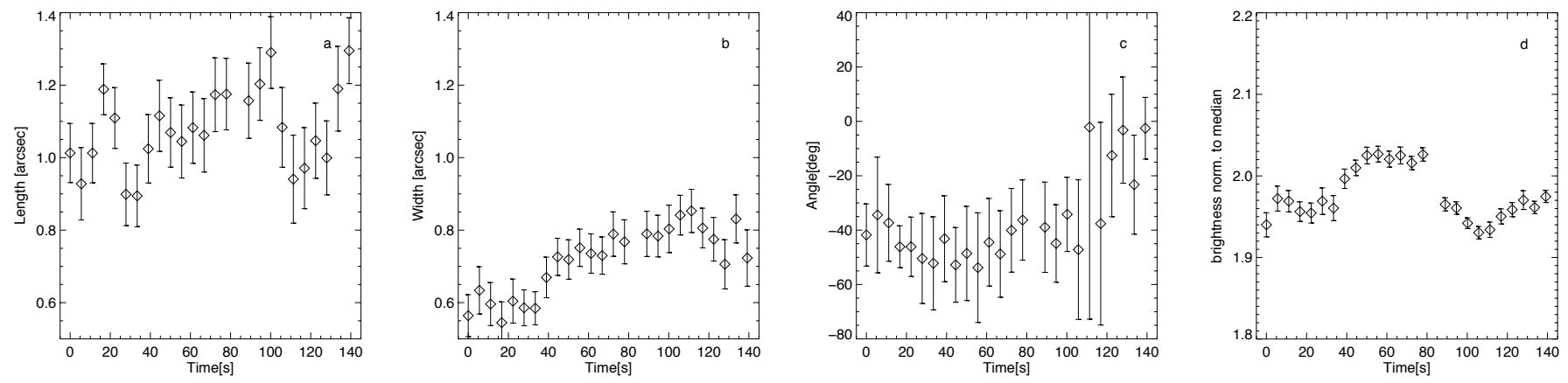

Fig. 5. Temporal variation of the properties of one loop-like feature. This plot is for feature B as marked in Fig. 3. The properties of the other three features are shown in Figs. A.1 to A.3. These data are based on the ellipse fits of the features: a) the major axis representing the length; b) the minor axis the width; c) the angle of the major semi-axis with the E-W direction for the angle; and d) the intensity integrated over the ellipse for the structure brightness. The time is with respect to the first full-frame Hi-C image at 18:53:11 UT. See Sect. 3.1.

idea that no systematic effects cause this result. In general, these structures exhibit random motions, which are consistent with the motion of the small-scale magnetic loops arching over granules discussed in the introduction. In particular, this horizontal velocity is consistent with observations of flux emergence on granular scales. As shown by Martínez González \& Bellot Rubio (2009), in those cases, footpoints can be separated by approximately $2^{\prime \prime}$ within four minutes (see their Fig. 2), which would be approximately $6 \mathrm{~km} \mathrm{~s}^{-1}$. Thus, the average velocity of the features we see is approximately comparable to average horizontal photospheric motions under small-scale flux-emergence conditions.

In general, while the structures move, they also (slightly) change their length and width. This is illustrated in Figs. 5a, b for feature B (for the other features see Figs. A.1 to A.3). The typical length and width of the loop-like structures are $1 \mathrm{Mm}$ and $0.5 \mathrm{Mm}$, respectively, with a typical aspect ratio of approximately two. Strictly speaking, the footpoint distance of approximately $1 \mathrm{Mm}$ is only representative of the loop length if it is a low-lying (loop) structure (which we implicitly assume here). If it were a semi-circular loop, its length would be $\pi / 2$ times the footpoint distance, that is, some $50 \%$ longer.

Besides the horizontal motion of the features as a whole (Fig. 4), we also follow the motion of their endpoints (viz. footpoints) relative to their center. This is done by measuring the angle of the fitted ellipse with respect to the E-W direction. As an example, Fig. $5 c$ shows the change of this angle during the Hi-C observation for feature B (again, other features in Figs. A.1 to A.3). Because of the considerable uncertainties in determining the angle (because the ratio of length to width is not big), the variation of the angle is almost within the error bars. Nevertheless, in three of the four cases we see a gradual rotation of the loop feature, with a difference between the maximum and minimum angles of approximately $\alpha \approx 40^{\circ}$ (expect the case in Fig. A.1c). While the question remains as to the significance of this value, we can at least estimate this as an upper limit. Considering the length of approximately $1 \mathrm{Mm}$, this angle corresponds to a rotational component of the motion of approximately $\sin \left(40^{\circ}\right) \times 1 \mathrm{Mm} \approx 0.6 \mathrm{Mm}$. Thus the upper limit of the speed of the footpoints during the $140 \mathrm{~s}$ of the Hi-C full-frame data is approximately $4 \mathrm{~km} \mathrm{~s}^{-1}$. This is approximately consistent with the velocity of magnetic concentrations in the intergranular lanes derived from magnetic bright points (Jafarzadeh et al. 2014, their Fig. 2) and supports the interpretation that the footpoints are rooted in flux tubes in intergranular lanes.

Finally, we verified the variability of the brightness in time. For feature B this is shown in Fig. 5d. There is a variation of approximately 5\% (for other features, even up to $15 \%$ ) of the brightness over approximately $100 \mathrm{~s}$. This variation of the brightness is significant in two respects. Firstly, this variability is approximately four times larger than the average error in brightness (taking into account the photon noise and the error in the ellipse fitting shown as bars in the plots). Secondly, this variation of $5 \%$ to $15 \%$ is also significant when considering that the contrast of the loop-like features above the background of the plage is only some $20 \%$ (see end of Sect. 3.1). This implies, that the change of the emission from the loop-like feature alone would change by $50 \%$ to almost $100 \%$ during the observation time. Still, the loop-like features are visible just above the background throughout the full Hi-C observation sequence of approximately two minutes. Naturally we can not draw a final conclusion on the lifetime of the loop-like features because the length of the time series of the full-frame $\mathrm{Hi}-\mathrm{C}$ images is limited, but at least we can say that the lightcurve is consistent with a feature lifetime of two minutes or more. For the other features, the situation is less clear (Figs. A.1 to A.3). One feature shows almost no intensity variation, while the other two are caught while the intensity decreases during the observation time. We can thus conclude that the features probably have a lifetime of a few minutes or more.

Summarising these findings, the observed features show motions and lifetimes consistent with being anchored in magnetic concentrations in intergranular lanes. This would be consistent with the loop-like features being indeed miniature hot loops spanning one granule, and them being the result of rising magnetic flux tubes of the type reported by Ishikawa et al. (2010), for example. Furthermore their properties in terms of length, width and lifetime are comparable to the transition region loops at $0.1 \mathrm{MK}$ reported by Hansteen et al. (2014), therefore it might be that the features we see here are a coronal temperature version of those transition region loops.

However, based on the above information, it could still be that we see only one footpoint of a longer loop, similar to the scenarios discussed in the introduction (cf. Fig 1). For this, we discuss their relation to the magnetic field (Sect. 4) and the thermal structure (Sect. 5).

\section{Relation to photospheric field}

For an investigation of how the loop-like features relate to the magnetic field, we carefully aligned the Hi-C images with the HMI magnetograms (for details of the alignment see Appendix B). The accuracy of the alignment of the HMI magnetogram with the Hi-C image is within less than half a pixel 


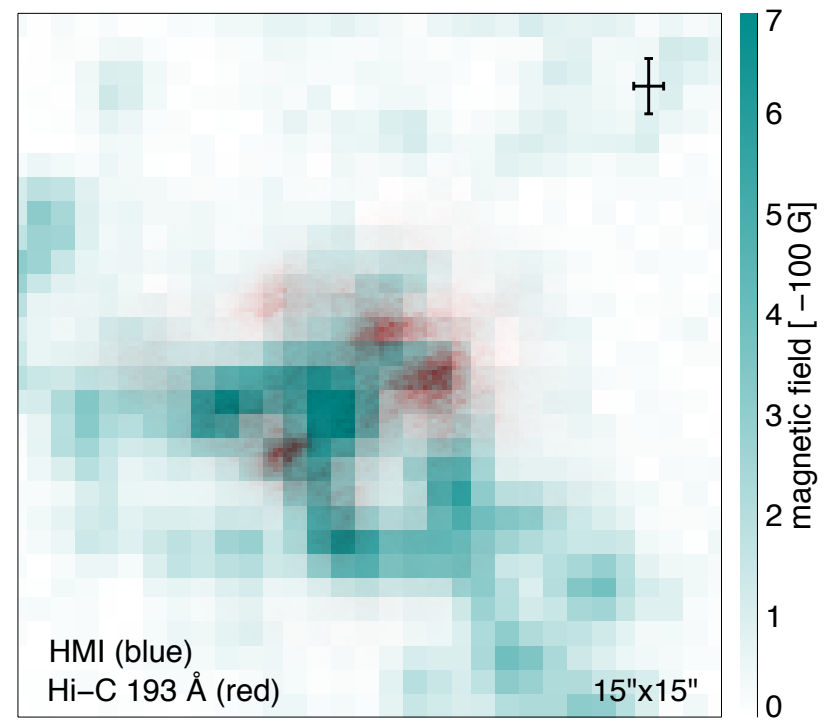

Fig. 6. Aligned composite of photospheric magnetogram and coronal emission. In the same field-of-view as in Fig. 3 we plot the magnetogram on a blue scale and overlay the coronal image from $\mathrm{Hi}-\mathrm{C}$ in red. The magnetogram in this field-of-view contains only one single magnetic polarity. The Hi-C image is the same as in Fig. 3, but on a non-linear colour scale to emphasise the loop-like features. See Sect. 4.

of HMI, at approximately $0.2^{\prime \prime}$. The result of the alignment is displayed as a composite in Fig. 6 showing the magnetogram along with the Hi-C image of coronal plasma over-plotted in red.

Within the sensitivity and resolution of HMI, only one polarity is seen in the magnetogram of this plage region, as noted already by Peter et al. (2013) in their study of this same region. In the field-of-view in Fig. 6 not a single pixel of the HMI magnetogram shows an opposite polarity. However, this does not exclude the presence of small parasitic opposite polarities. At higher spatial resolution and magnetic sensitivity, one might well see opposite polarities not visible to HMI. For example, data from the Imaging Magnetograph eXperiment (IMaX; Martínez Pillet et al. 2011) on the Sunrise balloon telescope (Solanki et al. 2010), at much higher resolution and sensitivity than HMI, clearly show small-scale magnetic patches of opposite polarity close to a largely unipolar region (e.g. Wiegelmann et al. 2013, their Fig. 2) that would not be detectable by HMI. Unfortunately, as discussed in Sect. 2, no observations of higher resolution or sensitivity are available for this region of interest.

The comparison of the Hi-C image and the magnetogram in Fig. 6 shows that typically at least part of the loop-like feature is located above a concentration of magnetic field. According to the scenarios in Fig. 1 this would support the theory that one (or the only) footpoint is rooted in the photospheric magnetic field concentration. Because of the arguments concerning weak, small and opposite-polarity patches, the missing detection of HMI of a possible second footpoint cannot be used as an argument against the small-scale loop scenario. Therefore this discussion of the relation of the magnetic field to the emission seen in $\mathrm{Hi}-\mathrm{C}$ must remain indecisive and we must rely on the analysis of the thermal structure at and around the plage region hosting the small looplike features.

\section{Relation to thermal structure}

There is a significant difference between a miniature coronal loop and the moss emission that might also appear as an elongated structure (Figs. 1a, b): by its very nature in the vicinity of the moss structure there should be some signature of the hot loop that is connected to the moss region, while for an isolated miniature loop one would not expect that hot component of the emission. To address this difference, we investigate the differential emission measure (DEM; Sect.5.1) and the X-ray emission (Sect. 5.2) from the plage region hosting the small loop-like features and compare it to a moss area.

\subsection{Differential emission measure (DEM)}

We perform a DEM analysis in the vicinity of the loop-like features and compare this to a moss region that has been analysed before (Testa et al. 2013) as well as to quiet regions without notable emission in the $193 \AA$ band for a blind test, all in the Hi-C field-of-view. The DEM provides some information on how the plasma is distributed in temperature throughout the atmosphere (along the line-of-sight) and is defined as

$D E M=n_{e}^{2}\left(\frac{\mathrm{d} T}{\mathrm{~d} h}\right)^{-1}$.

Here $n_{\mathrm{e}}$ is the electron density, $T$ the temperature, and $h$ the height along the line-of-sight. Being a function of $T$, the DEM is a measure for how much plasma is present at temperatures where the EUV (and X-ray) emission is originating. Hi-C alone can not be used to calculate the DEM because it provides only one wavelength band. In contrast, AIA includes a sufficient number of bands spanning the typical temperatures in the corona, albeit at a significantly lower spatial resolution. So we use AIA imaging data (see Table 1) to perform an inversion resulting in maps of the DEM over the covered temperature range. This analysis is based on a set of near-simultaneous images obtained between 18:53:56 and 18:54:06, one in each channel. In our analysis we took into account photon noise and readout noise ${ }^{2}$.

There are numerous publicly available methods to perform this inversion, and we decided to use the regularised DEM inversion by Hannah \& Kontar (2012) for its robustness.

To obtain DEM curves for each region-of-interest (see boxes $1-9$ in Figs. $2 \beta, \gamma$ ) we calculated the average intensity in each region-of-interest for each AIA channel. The inversion procedure as described by Hannah \& Kontar (2012) uses these AIA count rates as input and provides the DEM as a function of temperature, including error estimates for the temperature and the DEM (shown as a cross in Fig. 8). One contribution to the errors in the DEM are the uncertainties in the count rates of the AIA channels (with counts typically ranging from $50 \mathrm{DN} / \mathrm{pixel} / \mathrm{s}$ in the weak channels (e.g. $94 \AA$ ) to a few thousand DN/pixel/s in the stronger channels (e.g. $193 \AA$ ). However, the errors returned by the procedure also include uncertainties of the method and the width of the contribution functions of the AIA channels in temperature (for details see Hannah \& Kontar 2012).

The maps of the DEM in the vicinity of the small loop-like structures (Fig. 7) show a clear signature of an enhanced DEM at temperatures around $1.5 \mathrm{MK}(\log T=6.2$; here and in the following, all $\log T$ values refer to $T$ in units of $\mathrm{K}$ ). This simply

\footnotetext{
2 To calculate the photon noise and the read noise of the SDO/AIA images we use the procedure aia_bp_estimate_error available in SolarSoft (http: //www . lmsal. com/solarsoft/).
} 


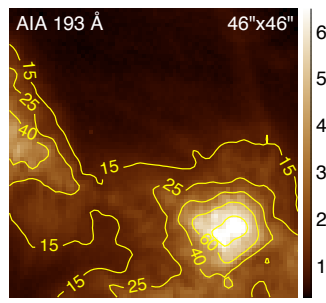

$\log \mathrm{T}=6.1$

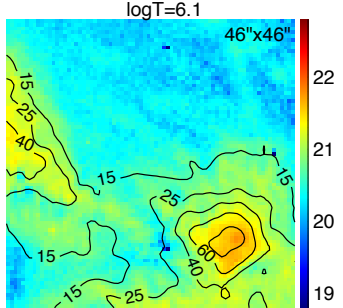

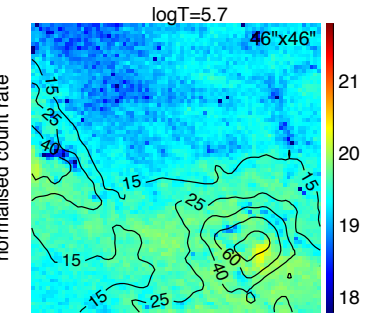

$\log T=6.2$

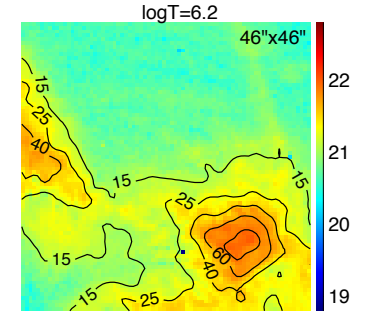

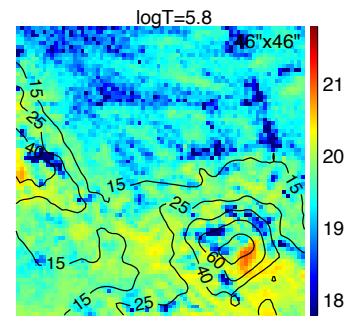

$\log \mathrm{T}=6.3$

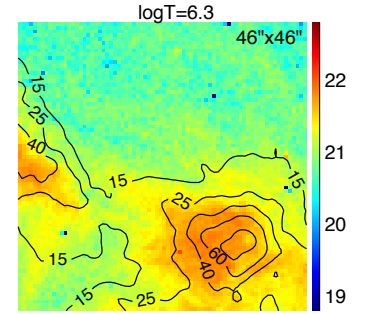

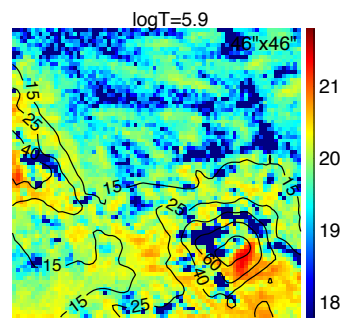

$\log T=6.4$

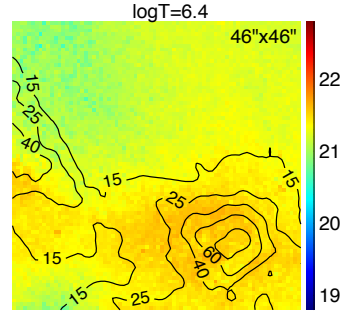

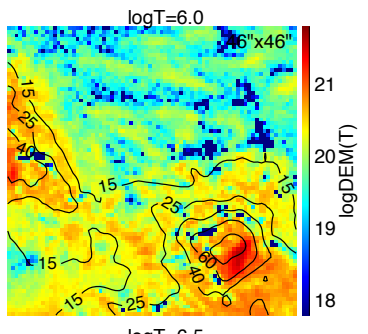

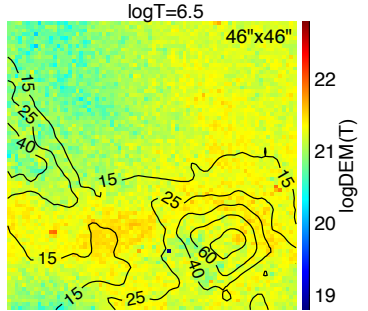

Fig. 7. Coronal image and thermal structure. The top left panel shows the AIA image at $193 \AA$. The other panels display spatial maps of the differential emission measure (DEM) at different temperatures $T$ labeled by $\log T[\mathrm{~K}]$. All panels show the same field-of view that is identical to Fig. $2 \beta$. The plage region with the miniature loop-like structures is in the bottom-right part. For a better comparison between the panels we add contour lines of the brightness in the AIA $193 \AA$ channel to all the panels. The numbers with the levels denote the percentage of the peak $193 \AA$ brightness in this field-of-view. All DEM maps have the same dynamic range of $10^{4}$ although their lower (and upper) limits change to best represent the DEM structures. See Sect. 5.1.

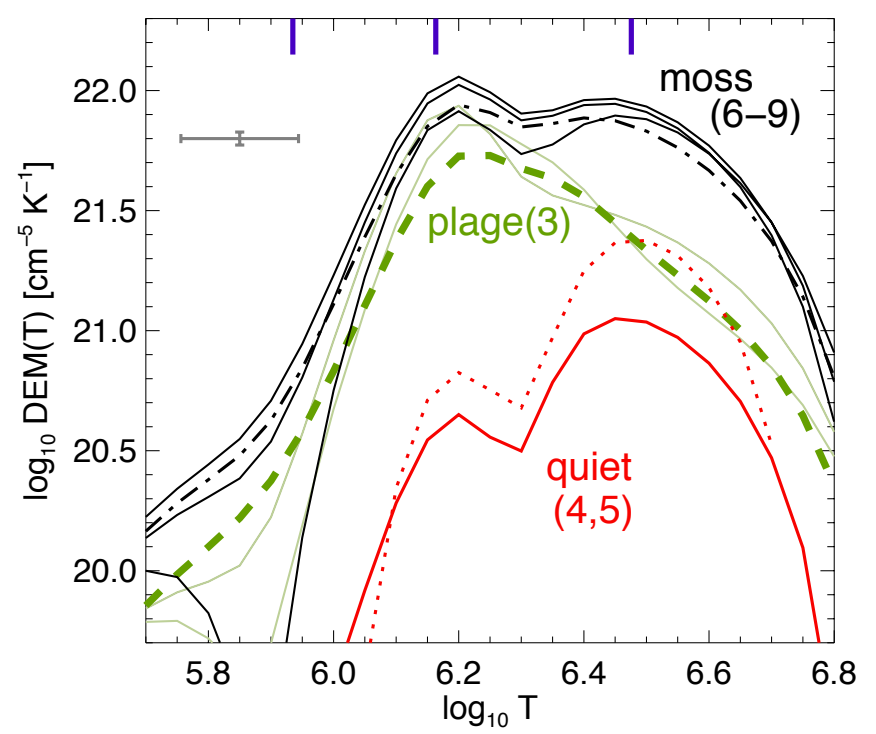

Fig. 8. Differential emission measure (DEM) distribution for different structures. The labelling of the lines corresponds to the regions marked in Figs. $2 \beta, \gamma$. The green lines represent two individual small loop-like features (thin green; 1,2 ) and an average covering the whole plage area hosting the small loop features (thick dashed green; 3 ). For comparison the red lines are the inversions for the quiet regions $(4,5)$. The black lines show the DEM in the moss areas (6-8), with the thick dashed line for the average over the larger moss region (9). The error bars (grey) present the average uncertainties for all curves in $\log T$ range from 6.0 to 6.7. The three blue markers at the top axis indicate the temperature of the peaks of the DEM components in coronal holes, quiet Sun, and active region according to Landi \& Feldman (2008). See Sect. 5.1.

reflects the enhanced emission we see in the Hi-C passband. In particular, the DEM maps do not show any significant signal at higher temperatures.

To emphasise this result from the spatial maps, we check the DEM curves as a function of $T$ for selected regions (Fig. 8). In the areas directly above two of the loops (regions 1 and 2 in Figs. $2 \beta$ and 3 ) as well as in a larger area encompassing all the small loop-like features in the plage region (region 3 in Figs. $2 \beta$ and 3) we see a clear peak at approximately $\log T=6.2$. The drop to lower and higher temperatures is comparably sharp. In particular, the drop to higher temperatures distinguishes the loop-like features from the moss area (see below). As expected, the DEM from the plage region is significantly higher than in sample regions covering the quiet regions (see Fig. 8). In particular, also region 5 in Fig. $2 \beta$, that is located at a faint longer loop seemingly connecting the plage area with the main part of the active region to the North, does not show any enhanced DEM at higher temperatures, at least not higher than the DEM curves for the plage regions 1 to 3 . This is why we labelled this region 5 as "quiet", as well, in Fig. 8.

To confirm that this technique would detect the presence of hot plasma in moss regions, we checked the moss region in the Hi-C field-of-view that has been investigated already by Testa et al. (2013). These are regions 6 to 9 in Fig. $2 \gamma$. The DEM curves of these regions in Fig. 8 are distinctively different from the loop-like features in the plage. While the DEM of the moss regions is comparable around $\log T=6.2$, where the plage region peaks, only the moss regions show a clear enhancement of the DEM up to at least $\log T=6.6$.

Therefore we conclude that there is no hot plasma present in the vicinity of the loop-like features. At least not to the same extent as in moss regions. To further verify this, we also investigated the X-ray emission.

\subsection{X-ray observations}

Including X-ray observations can help to relax the limitation of AIA in terms of temperature coverage. While AIA alone can provide the DEM only up to approximately $\log T=6.6$ or 6.7 ( $\approx 5 \mathrm{MK}$; cf. Fig. 8), XRT observations cover a broad range of temperatures centered at almost $10 \mathrm{MK}$ (cf. Table 1). In principle one could include X-ray observations in the DEM inversion (e.g. Cheung et al. 2015. Here we choose the more straight 

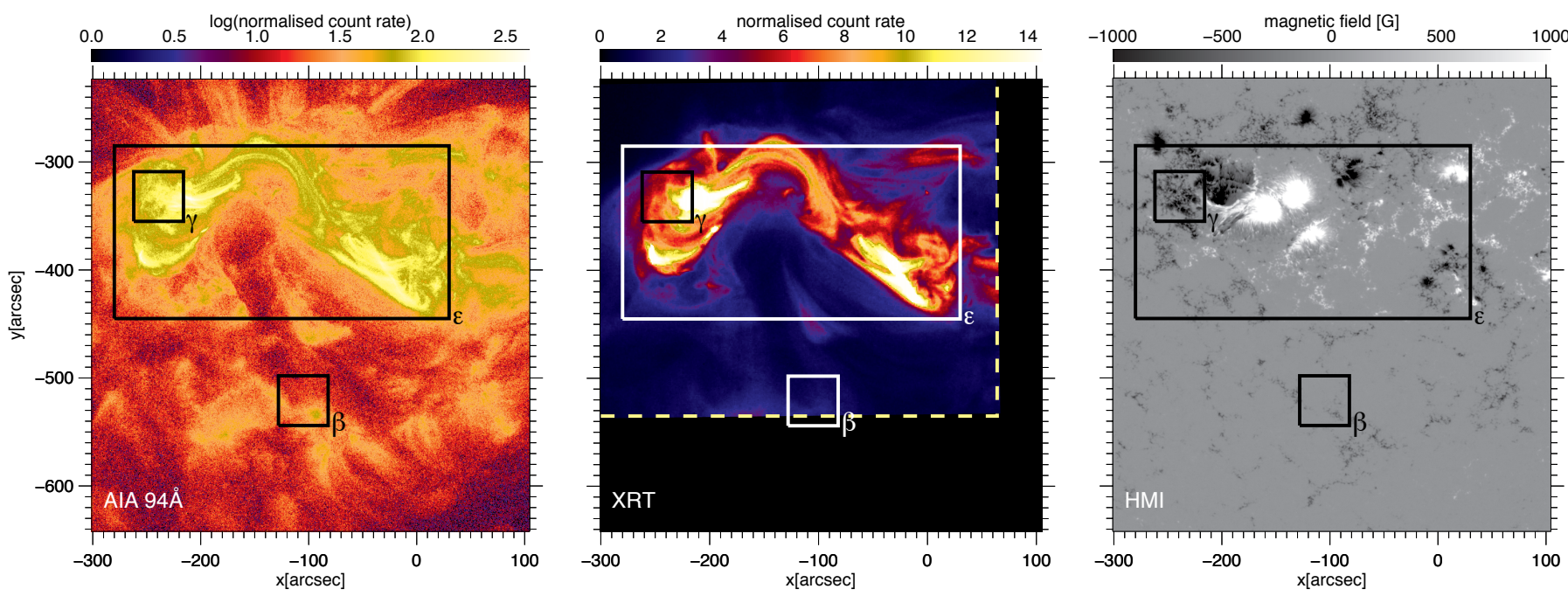

Fig. 9. Emission from hot plasma and underlying magnetic field. The left two panels show the emission in the AIA channel at $94 \AA$ and the Ti-poly channel of XRT on Hinode. The XRT image is taken during the Hi-C flight (at 18:54:48 UT). The right panel shows the HMI magnetogram. All panels cover the full field-of-view of the Hi-C data (cf. large rectangle in Fig. $2 \alpha$ ). The inbox $\beta$ indicates the region with the plage area hosting the small loop-like features as also displayed in Fig. $2 \beta$. The rectangle $\varepsilon$ outlines the area covering hot coronal loops and moss as investigated by Testa et al. (2013). In their paper they display this region $\varepsilon$ rotated counter-clockwise by $90^{\circ}$ The moss area displayed in Fig. $2 \gamma$ and marked here also by $\gamma$ roughly corresponds to the moss region M3 of Testa et al. (2013), their Fig. 2. The yellow dashed line in the XRT image marks the edge of the XRT field-of-view. See Sect. 5.2.

forward way and study the X-ray images directly. This provides information if a hot component of a loop rooted in the loop-like features is present that might not be revealed by AIA. The DEM, as derived from AIA in the plage region hosting the small looplike features, drops steeply above $\log T=6.3(\approx 2 \mathrm{MK})$. The response of the Ti-Poly filter of XRT drops by a factor of (only) approximately 15 from its peak (at $9 \mathrm{MK}$ ) to $2 \mathrm{MK}$ (Golub et al. 2007). Therefore, XRT is well suited to test if there is a hot component of plasma that would go unnoticed by the DEM analysis of AIA. The hot emission from X-rays in the Hi-C field-of-view as seen by XRT on Hinode is displayed in Fig. 9. For comparison we show the AIA $94 \AA$ channel that reveals comparably hot plasma (cf. Table 1), hotter at least than $2 \mathrm{MK}$ and colder than 12.5 MK. In this context we used the AIA $94 \AA$ channel (together with AIA $193 \AA$ ) simply for aligning XRT with Hi-C.

One problem concerning the XRT data during the Hi-C flight is that they do not fully cover the whole field-of-view of Hi-C. In particular, they only partially cover the plage region hosting the loop-like features. Still, the XRT image shown in Fig. 9, which is taken during the Hi-C flight, fully covers the region between the negative magnetic polarity of the plage region and the positive polarity in the main part of the active region to the North. This is clear from the comparison to the HMI magnetogram in Fig. 9. Therefore XRT covers the region where one would expect the hot loops that might be rooted in the plage region ( $\beta$ in Fig. 9).

In the space covering the connection from the plage area with the small loop-like features to the main part of the active region, there is no significant X-ray emission visible (Fig. 9). While we see clear hot loops in X-rays in the main part of the active region that contains the moss regions already studied by Testa et al. (2013), such X-ray emission is not related to the plage region ( $\beta$ in Fig. 9). In fact, the region North of the plage region, where the magnetic connection would be expected, is particularly dark in X-rays. This extends the temperature range of the missing hot plasma related to the plage region with the small loop features and highlights the lack of significant amounts of plasma present at higher temperatures from above $2 \mathrm{MK}$ up to $10 \mathrm{MK}$.
With these considerations on the thermal structure based on the DEM and the X-ray emission, we conclude that it is unlikely that the loop-like features are the footpoints of hot (and dense) loops. Therefore, we exclude the moss scenario shown in Fig. 1b and discussed in the introduction.

\section{Discussion}

The above discussion of the thermal structure suggests that the loop-like features are not the moss-like footpoints of hot dense loops. This argument is based on the absence of a significant amount of hot plasma that would fill the related loop. Still, one could speculate that the scenario in Fig. 1c could apply; namely that the small loop-like features are at the footprints of larger (hot) but dilute loops (cf. Sect. 1). Heat input from below could heat the lowermost part, but leave the upper part of the loop unaffected. In this case, the longer loops would not be visible in $\mathrm{X}$-rays or leave a signature in the DEM at high temperatures, while we still see the compact loop-like features appearing at their footpoints. In principle, this would be consistent with the thermal structure we discussed in Sect. 5.

However, there are theoretical arguments against this interpretation of the small loop-like features being footpoints of long dilute loops. To illustrate this we conduct a Gedanken experiment: we assume that along a magnetic fieldline with a length of several tens of $\mathrm{Mm}$ close to the footpoint exists a dense structure at approximately $1.5 \mathrm{MK}$ with a length of approximately $1 \mathrm{Mm}$ along the fieldline. Above that, the loop is hotter but very dilute ${ }^{3}$ This structure would be, in general, consistent with our observation (according to the scenario in Fig. 1c). We

\footnotetext{
3 Alternatively, the upper part of the loop could also be cool, say at only $10^{4} \mathrm{~K}$. Because the Barometric scale height at low temperatures (at $10^{4} \mathrm{~K}$ it would be only $500 \mathrm{~km}$ ) is small compared to the loop length, also in this case the loop would be at very low density in its upper part. Therefore, for a cool upper part of the loop the arguments would be the same as for a dilute hot upper part discussed here, and our conclusion would be identical.
} 
would see an elongated feature with a length of $1 \mathrm{Mm}$ and with a peak of the DEM at approximately $\log T \approx 6.2$ or $1.5 \mathrm{MK}$ (cf. Fig. 8), but no X-ray emission above, because the hot part of the loop is not dense enough. However, the thermal stratification assumed for this setup would not be stable over the time of one to two minutes when we observe the features during the Hi-C flight. The dense plasma at a temperature of $\log T \approx 6.2$ or 1.5 MK has a pressure scale height of approximately $75 \mathrm{Mm}$ (or 0.1 solar radii). With no (significant amount of) dense plasma above it, the dense $1.5 \mathrm{MK}$ plasma would quickly expand into the upper part of the loop, similarly to an expansion into vacuum. Expansion into vacuum happens at approximately the adiabatic sound speed, which for plasma at $1.5 \mathrm{MK}$ is approximately $185 \mathrm{~km} \mathrm{~s}^{-1}$. Even when assuming an expansion speed much less than that, $100 \mathrm{~km} \mathrm{~s}^{-1}$, for example, within the time-scale during which these structures exist (and we observe them), that is, approximately $100 \mathrm{~s}$, they should have expanded by approximately $10 \mathrm{Mm}\left(=100 \mathrm{~km} \mathrm{~s}^{-1} \times 100 \mathrm{~s}\right)$. This would be a distance much larger than the size of the structures, an expansion we should clearly see (e.g. in the form of a jet). This contradicts our initial assumption of 1.5 MK dense plasma below a hot dilute loop, because we do not see any significant expansion of the small looplike features. This leads us to the conclusion that they cannot be the footpoint regions of large dilute loops.

This leaves only one scenario, namely that the small looplike features are indeed tiny versions of hot coronal loops, as depicted in Fig. 1a. The main argument against this could be that in the HMI magnetograms we see only one single polarity in the plage region hosting these structures, as already pointed out by Peter et al. (2013). However, as discussed in Sect. 4, these HMI observations of only one single polarity do not rule out the possibility of small-scale opposite polarities, which is because of the limited sensitivity and spatial resolution; instruments with higher resolution and sensitivity have seen such small-scale opposite polarities (cf. Sect. 1), even though no such observations are available during the $\mathrm{Hi}-\mathrm{C}$ flight for the area we investigate.

There is also observational evidence for small loops at coronal temperatures through data from AIA; albeit those loops are significantly longer than the miniature loops we report here. Wang (2016) finds short coronal loops in AIA $171 \AA$ observations in plage regions that have footpoint distances from $3 \mathrm{Mm}$ to $5 \mathrm{Mm}$. This implies that their lengths are of the order of $5 \mathrm{Mm}$ to $10 \mathrm{Mm}$, depending which loop geometry is assumed. These loops also occur in plage regions that appear unipolar in HMI, and Wang (2016) uses this as indirect evidence for small-scale opposite polarity magnetic field hidden to HMI. In some way, the small loops in plages found recently by Wang (2016) could be considered as longer versions of the structures we study here in some detail and were first reported by Peter et al. (2013). In terms of modelling, it would be interesting to see what onedimensional loop models would predict for very short loops that are heated to $1.5 \mathrm{MK}$ or more, if that is possible. The models by Sasso et al. (2012) produce transition region loops with temperatures well below $1 \mathrm{MK}$ only. If, in those models, the energy input is increased, the loops might reach higher temperatures, and it would be interesting to see if they turn out to be stable or highly intermittent.

\section{Conclusions}

We investigated small loop-like features in the solar corona seen in the $193 \AA$ channel of Hi-C. They have typical lengths of $1 \mathrm{Mm}$ and lifetimes of some $100 \mathrm{~s}$. They appear in a plage or enhanced network region at the periphery of an active region. The analysis of the morphology of these structures in terms of horizontal motions and lifetime suggests a close relationship with the granular motions in the photosphere (Sect. 3.2). One end of the loop-like features is rooted in a magnetic field concentration as seen by HMI. The limited resolution and sensitivity of HMI prevents definite conclusions if the other end of the feature is rooted in an opposite magnetic polarity (Sect. 4).

The analysis of the thermal structure of the loop-like features (Sect. 5) shows that they cannot be the footpoints of hot dense loops, as is the case for moss (cf. Fig. 1b). This is supported by a DEM analysis (Sect. 5.1) as well as by the direct X-ray observations and a comparison to actual moss structures (Sect. 5.2). Theoretical arguments can be made that the loop-like features are also not at the footpoints of (hot) dilute loops (cf. Fig. 1c) heated from their footpoints, because then we would expect to observe expanding (jet-like) features, which we do not see (Sect. 6).

This discussion leaves us interpreting the loop-like features as actual miniature coronal loops (cf. Fig. 1a). These would span just a single granule and connect a magnetic concentration in an intergranular lane with a feature of opposite- polarity on the other side of the granule. That HMI does not see a parasitic opposite polarity does not exclude this scenario, because high-resolution photospheric magnetic field observations (e.g. Martínez González \& Bellot Rubio 2009; Wiegelmann et al. 2013), as well as indirect evidence from coronal structures (Wang 2016), provide evidence for the existence of such smallscale opposite polarities (Sects. 4 and 6).

These miniature loops might well be related to small-scale flux emergence events, where small magnetic fluxtubes break through the photosphere (e.g. Ishikawa et al. 2010). Clear evidence for such short $(1 \mathrm{Mm})$ loops reaching transition region temperatures (i.e. approximately $0.1 \mathrm{MK}$ ) has been reported recently using IRIS data (Hansteen et al. 2014). It does not seem unrealistic that, in cases of stronger heating, such loops might reach higher coronal temperatures of more than $1 \mathrm{MK}$. The transition region loops are visible by IRIS because of its high spatial resolution of approximately $0.3^{\prime \prime}$. To see such miniature loops in the corona, one needs comparable spatial resolution, which is not available with the current workhorse of coronal imaging, AIA, but is made available by the rocket experiment $\mathrm{Hi}-\mathrm{C}$ that we used here. For this (and for other reasons) we hope for a reflight of Hi-C.

Acknowledgements. We very much appreciate the valuable comments of the anonymous referee. We would like to thank Philippe Bourdin for advice on the fitting of ellipses. Thanks are also due to Leping Li and Chen Nai-Hwa for helpful suggestions. This work was supported by the International MaxPlanck Research School (IMPRS) for Solar System Science at the University of Göttingen.

\section{References}

Berger, T. E., De Pontieu, B., Schrijver, C. J., \& Title, A. M. 1999, ApJ, 519, L97

Boerner, P., Edwards, C., Lemen, J., et al. 2012, Sol. Phys., 275, 41

Bray, R. J., Cram, L. E., Durrant, C., \& Loughhead, R. E. 1991, in Plasma Loops in the Solar Corona (Cambridge, UK: Cambridge University Press)

Brown, D. S., Parnell, C. E., Deluca, E. E., Golub, L., \& McMullen, R. A. 2001, Sol. Phys., 201, 305

Centeno, R., Socas-Navarro, H., Lites, B., et al. 2007, ApJ, 666, L137

Cheung, M. C. M., Boerner, P., Schrijver, C. J., et al. 2015, ApJ, 807, 143

Cirtain, J. W., Golub, L., Winebarger, A. R., et al. 2013, Nature, 493, 501

De Pontieu, B., Title, A. M., Lemen, J. R., et al. 2014, Sol. Phys., 289, 2733

Dere, K. P. 2008, A\&A, 491, 561

Dravins, D. 1975, Sol. Phys., 40, 53 
Feldman, U. 1983, ApJ, 275, 367

Golub, L., Krieger, A. S., Silk, J. K., Timothy, A. F., \& Vaiana, G. S. 1974, ApJ, 189, L93

Golub, L., Deluca, E., Austin, G., et al. 2007, Sol. Phys., 243, 63

Hannah, I. G., \& Kontar, E. P. 2012, A\&A, 539, A146

Hansteen, V., De Pontieu, B., Carlsson, M., et al. 2014, Science, 346, 1255757

Ishikawa, R., \& Tsuneta, S. 2009, in The Second Hinode Science Meeting.

Beyond Discovery-Toward Understanding, eds. B. Lites, M. Cheung,

T. Magara, J. Mariska, \& K. Reeves, ASP Conf. Ser., 415, 132

Ishikawa, R., Tsuneta, S., Ichimoto, K., et al. 2008, A\&A, 481, L25

Ishikawa, R., Tsuneta, S., \& Jurčák, J. 2010, ApJ, 713, 1310

Jafarzadeh, S., Cameron, R. H., Solanki, S. K., et al. 2014, A\&A, 563, A101

Kobayashi, K., Cirtain, J., Winebarger, A. R., et al. 2014, Sol. Phys., 289, 4393

Landi, E., \& Feldman, U. 2008, ApJ, 672, 674

Lemen, J. R., Title, A. M., Akin, D. J., et al. 2012, Sol. Phys., 275, 17

Lites, B. W., Leka, K. D., Skumanich, A., Martinez Pillet, V., \& Shimizu, T. 1996, ApJ, 460, 1019

Martínez González, M. J., \& Bellot Rubio, L. R. 2009, ApJ, 700, 1391
Martínez Pillet, V., Del Toro Iniesta, J. C., Álvarez-Herrero, A., et al. 2011, Sol. Phys., 268, 57

Morton, R. J., \& McLaughlin, J. A. 2014, ApJ, 789, 105

O’Dwyer, B., Del Zanna, G., Mason, H. E., Weber, M. A., \& Tripathi, D. 2010, A\&A, 521, A21

Peter, H. 2000, A\&A, 360, 761

Peter, H. 2001, A\&A, 374, 1108

Peter, H., Bingert, S., Klimchuk, J. A., et al. 2013, A\&A, 556, A104

Peter, H., Tian, H., Curdt, W., et al. 2014, Science, 346, 1255726

Reale, F. 2010, Liv. Rev. Sol. Phys., 7, 5

Sasso, C., Andretta, V., Spadaro, D., \& Susino, R. 2012, A\&A, 537, A150

Scherrer, P. H., Schou, J., Bush, R. I., et al. 2012, Sol. Phys., 275, 207

Solanki, S. K., Barthol, P., Danilovic, S., et al. 2010, ApJ, 723, L127

Testa, P., De Pontieu, B., Martínez-Sykora, J., et al. 2013, ApJ, 770, L1

Wang, Y.-M. 2016, ApJ, 820, L13

Warren, H. P., \& Winebarger, A. R. 2000, ApJ, 535, L63

Wiegelmann, T., Solanki, S. K., Borrero, J. M., et al. 2013, Sol. Phys., 283, 253

Winebarger, A. R., Cirtain, J., Golub, L., et al. 2014, ApJ, 787, L10 


\section{Appendix A: Properties of individual loop-like features}

In Sect. 3.2 we discussed the properties of the loop-like structures in terms of length, width, orientation and brightness. There we mainly concentrated on one single structure, namely feature B marked in Fig. 3. Here we add the corresponding properties for the three additional structures, A, C and D, as marked in Fig. 3. In the same format as used in Fig. 5 for the time evolution of the properties of feature B, we show here in Figs. A.1 to A.3 the properties of features A, C and D.
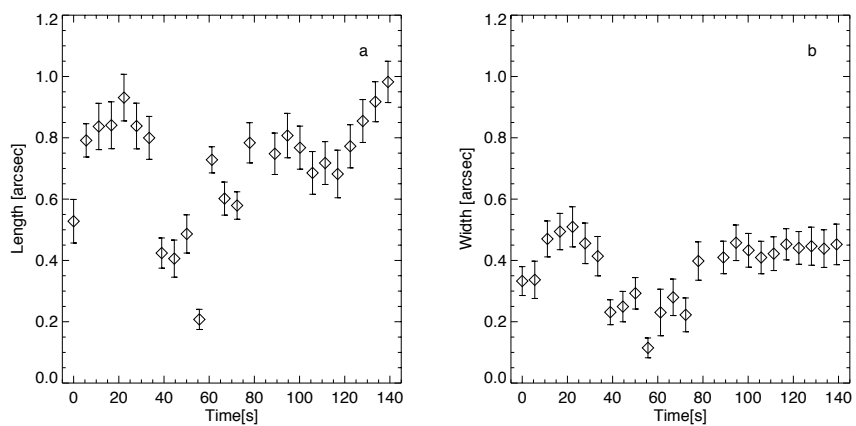

The properties of all four features are approximately similar, except for the evolution of the brightness. We could follow only feature B over a full life-cycle, that is, from an increase of the brightness until it faded away again within less than 2 min (cf. Fig. 5d). The three other features shown here are seen either only in the declining phase (Figs. A.1 and A.2) or show an almost constant brightness (Fig. A.3).

Fig. A.1. Properties of loop-like feature A marked in Fig. 3. Same format as Fig. 5.
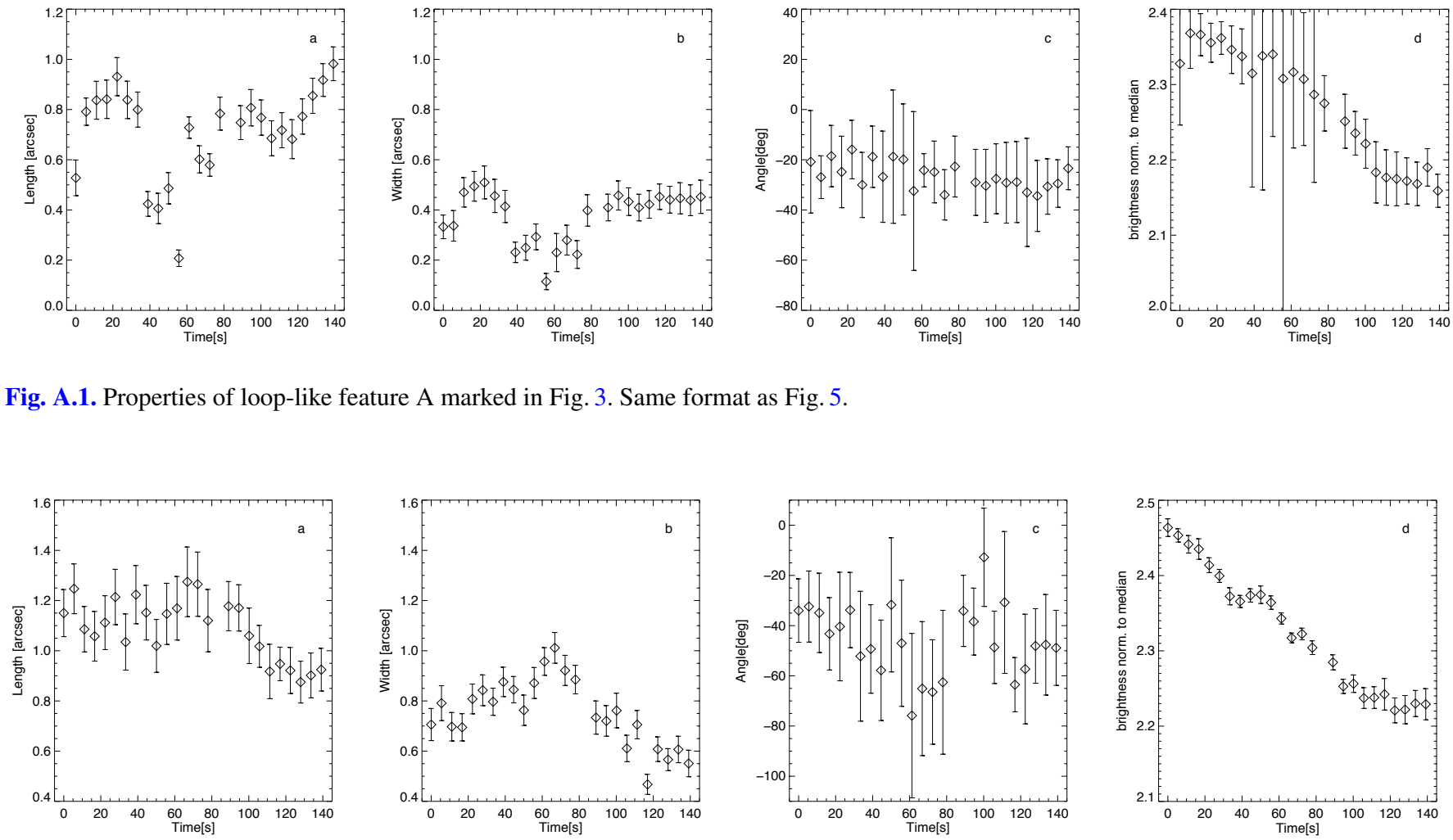

Fig. A.2. Properties of loop-like feature C marked in Fig. 3. Same format as Fig. 5.
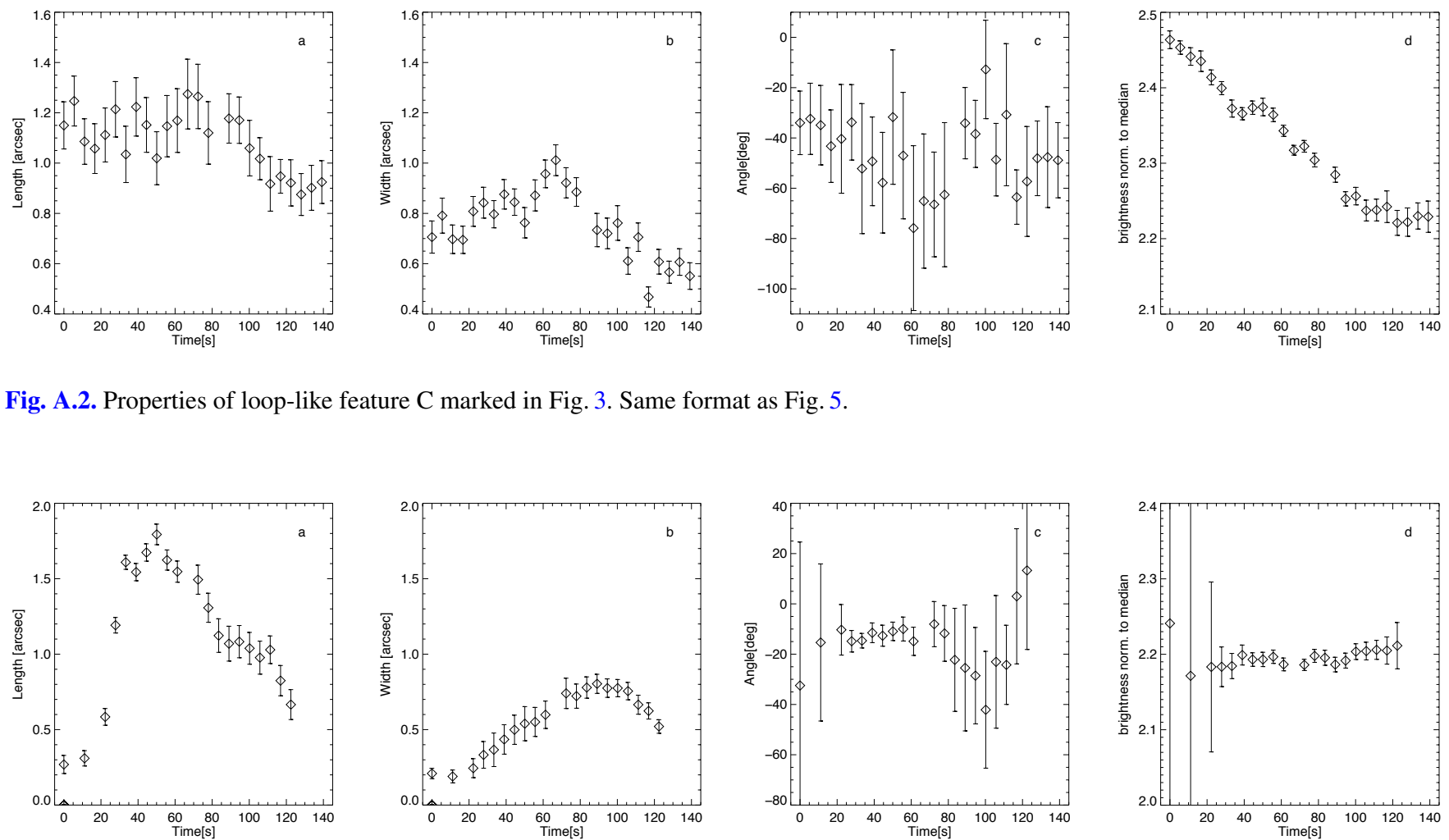

Fig. A.3. Properties of loop-like feature D marked in Fig. 3. Same format as Fig. 5. 


\section{Appendix B: Spatial alignment of $\mathrm{Hi}-\mathrm{C}$ with magnetogram}

To investigate the relation of the small loop-like coronal structures to the magnetic field in the photosphere we have to perform an alignment through different steps. The result of this alignment procedure is shown in Fig. 6 and discussed in Sect. 4. In this appendix we briefly describe the alignment procedure.

For the alignment chain we use a subregion of approximately $100^{\prime \prime} \times 100^{\prime \prime}$ with the plage region hosting the small loop-like structures at approximately the center. All images used for the alignment are scaled (through interpolation) to the pixel scale of $\mathrm{Hi}-\mathrm{C}$. We first align the $\mathrm{Hi}-\mathrm{C}$ image to the AIA image in the same $193 \AA$ channel. Because these images show the same coronal emission, only at different spatial resolution, this alignment is very reliable. The next step is to align the AIA $193 \AA$ image to the AIA $131 \AA$ channel. While the temperature of the peak contribution is quite different (cf. Table 1), both channels also have a significant contribution from lower (transition region) temperatures from $\log T=5.4$ to 5.6 (cf. Boerner et al. 2012). Therefore, the plage and enhanced network regions around our region-of-interest show many similar structures in the emission in the $193 \AA$ and the $131 \AA$ that provide a good basis for the alignment. This is also the case for the next step when aligning the AIA $131 \AA$ channel with the AIA $1600 \AA$ images that show the low chromosphere, because in both channels the emission from the
Table B.1. Accuracy of spatial alignment in solar $x$ and $y$ directions.

\begin{tabular}{ccc}
\hline \hline Images & $x\left[^{\prime \prime}\right]$ & $y\left[{ }^{\prime \prime}\right]$ \\
\hline HMI $\leftrightarrow$ AIA 1600 & 0.06 & 0.13 \\
AIA 1600 $\leftrightarrow$ AIA 131 & 0.10 & 0.24 \\
AIA 131 $\leftrightarrow$ AIA 193 & 0.13 & 0.18 \\
AIA 193 $\leftrightarrow$ Hi-C & 0.01 & 0.01 \\
\hline
\end{tabular}

(enhanced) network will dominate. In the final step we align the AIA $1600 \AA$ image with the HMI magnetogram. This alignment procedure is illustrated in Fig. B.1, where we show the respective images together with contour plots of the image in the next step of the alignment procedure.

For all alignment steps we use a cross-correlation technique to determine the misalignment and then shift the images with sub-pixel accuracy. We test the accuracy of the alignment through an alignment of the final aligned images. In Table B.1 we list the misalignment (separate for the solar $x$ and $y$ directions) of all alignment steps. Combined, these give a misalignment of $0.2^{\prime \prime}$ and $0.3^{\prime \prime}$ in the $x$ and $y$ directions, respectively. This alignment accuracy is of the order of the spatial resolution of Hi-C, or a fraction of a pixel of HMI or AIA, just as one would expect. This emphasises the fact that we can trust the spatial alignment between $\mathrm{Hi}-\mathrm{C}$ and the HMI magnetograms, which is the basis for the discussion on the relation of the small loop-like features to the magnetic field in Sect. 4. 

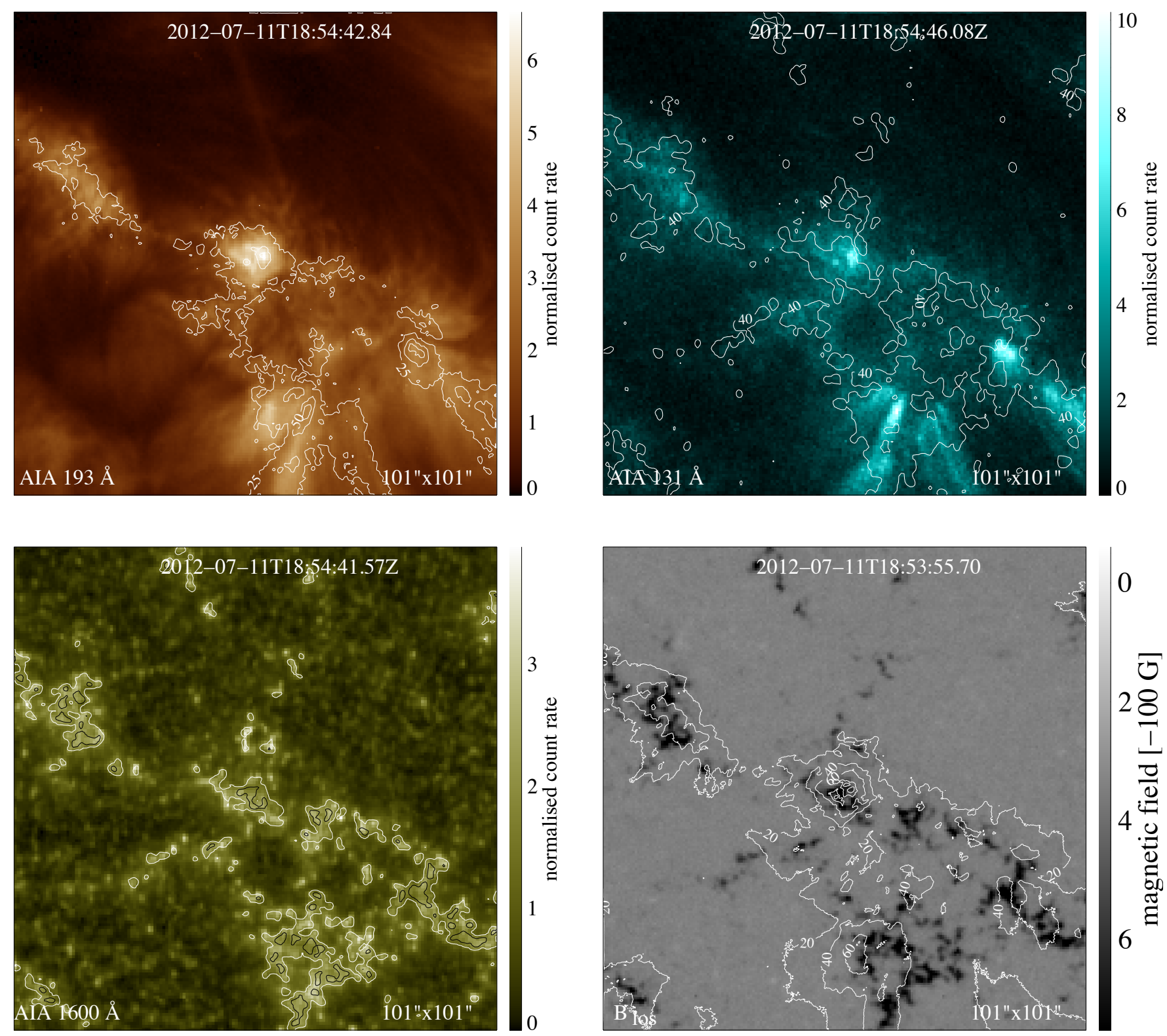

Fig. B.1. Illustration of the alignment procedure. The panels show the sequence of $101^{\prime \prime} \times 101^{\prime \prime}$ images of the alignment chain, that is, AIA $193 \AA \rightarrow$ AIA $131 \AA \rightarrow$ AIA $1600 \AA \rightarrow$ HMI magnetogram. In each image the contours of the respective image following in the chain are overplotted. The Hi-C $193 \AA$ image is aligned with the AIA $193 \AA$ channel and its contours are overplotted on the HMI magnetogram. The magnetogram contours are at $-100 \mathrm{G}$ (white) and $-300 \mathrm{G}$ (black). The other contours show the percentages of the maximum brightness in the respective channel (numbers with the contour lines). 